\title{
Meissner effect, Spin Meissner effect and charge expulsion in superconductors
}

\author{
J. E. Hirsch \\ Department of Physics, University of California, San Diego, La Jolla, CA 92093-0319
}

(Dated: May 23, 2011)

\begin{abstract}
The Meissner effect and the Spin Meissner effect are the spontaneous generation of charge and spin current respectively near the surface of a metal making a transition to the superconducting state. The Meissner effect is well known but, I argue, not explained by the conventional theory, the Spin Meissner effect has yet to be detected. I propose that both effects take place in all superconductors, the first one in the presence of an applied magnetostatic field, the second one even in the absence of applied external fields. Both effects can be understood under the assumption that electrons expand their orbits and thereby lower their quantum kinetic energy in the transition to superconductivity. Associated with this process, the metal expels negative charge from the interior to the surface and an electric field is generated in the interior. The resulting charge current can be understood as arising from the magnetic Lorentz force on radially outgoing electrons, and the resulting spin current can be understood as arising from a spin Hall effect originating in the Rashba-like coupling of the electron magnetic moment to the internal electric field. The associated electrodynamics is qualitatively different from London electrodynamics, yet can be described by a small modification of the conventional London equations. The stability of the superconducting state and its macroscopic phase coherence hinge on the fact that the orbital angular momentum of the carriers of the spin current is found to be exactly $\hbar / 2$, indicating a topological origin. The simplicity and universality of our theory argue for its validity, and the occurrence of superconductivity in many classes of materials can be understood within our theory.
\end{abstract}

PACS numbers:

\section{INTRODUCTION}

The Meissner effect [1] is the most fundamental property of superconductors. I argue that the Meissner effect is not accounted for by the conventional BCSEliashberg-London framework generally believed to explain all aspects of the superconductivity of conventional superconductors 2] (termed 'class 1' superconductors in Ref.[3]). Instead, I propose that superconductivity involves fundamental physics that is not described by conventional theory, namely: (i) superconductors expel negative charge from the interior to the surface[4]; (ii) it requires dominance of hole carrier transport in the normal state [5, 6]; (iii) it is driven by lowering of kinetic energy of the carriers [7, 8]; (iv) an electric field exists in the interior of superconductors [9], (v) a spin current exists near the surface, in the absence of applied external fields [10], and (vi) superconducting carriers reside in mesoscopic orbits of radius $2 \lambda_{L}[1]$, with $\lambda_{L}$ the London penetration depth. I argue that the Meissner effect, exhibited by all superconductors, cannot be accounted for unless the above listed effects also exist in superconductors.

\section{THE KEY PHYSICAL ELEMENTS}

Figure 1 shows three key aspects of the physics of superconductors within the theory discussed here. (a) The charge distribution in the superconductor is macroscopically inhomogeneous, with excess negative charge near the surface and excess positive charge in the interior. (b) Superfluid carriers reside in overlapping mesoscopic or- (a)

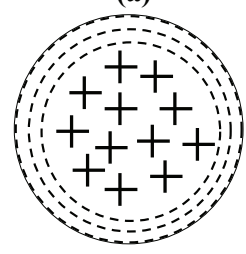

(b)
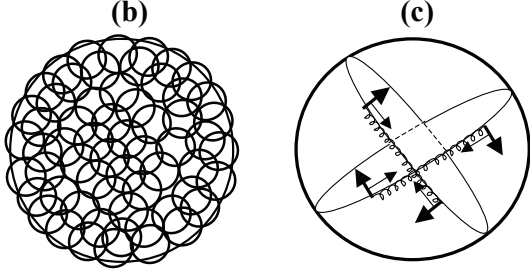

FIG. 1: Illustration of three key aspects of the physics of superconductors proposed here. (a) Superconductors expel negative charge from their interior to the region near the surface; (b) Carriers reside in mesoscopic overlapping orbits of radius $2 \lambda_{L}\left(\lambda_{L}=\right.$ London penetration depth); (c) A spin current flows near the surface of superconductors (the arrow perpendicular to the orbit denotes the direction of the electron magnetic moment).

bits of radius $2 \lambda_{L}$. (c) A macroscopic spin current flows near the surface of superconductors in the absence of applied external fields.

First, why does the Meissner effect imply an inhomogeneous charge distribution as depicted in Fig. 1(a)? Because in order for a Meissner current to be 'spontaneously' generated near the surface of superconductors in the presence of a magnetic field $B$ (in the $z$ direction), carriers have to move radially outward, to be deflected by the magnetic Lorentz force

$$
\vec{F}=\frac{e}{c} \vec{v} \times \vec{B}=\frac{e}{c} B\left(v_{r} \hat{\theta}-v_{\theta} \hat{r}\right) \equiv F_{\theta} \hat{\theta}+F_{r} \hat{r}
$$

in the azimuthal $(\hat{\theta})$ direction. In the absence of radial motion $\left(v_{r}=0\right), F_{\theta}=e B v_{r} / c=0$, there is no component of the force in the azimuthal direction and the 
spontaneous generation of an azimuthal Meissner current cannot be understood.

Second, why does the Meissner effect necessitate orbits of radius $2 \lambda_{L}$ ? The Larmor diamagnetic susceptibility of $n$ electrons per unit volume in orbits of radius $r$ for magnetic field perpendicular to the orbits is

$$
\chi_{\text {Larmor }}=-\frac{n e^{2}}{4 m_{e} c^{2}} r^{2}
$$

and takes the value $-1 / 4 \pi$, describing perfect diamagnetism, for

$$
r^{2}=\frac{m_{e} c^{2}}{\pi n e^{2}}
$$

from which we conclude that $r=2 \lambda_{L}$, with $\lambda_{L}$ the London penetration depth, defined by the standard equation [2]

$$
\frac{1}{\lambda_{L}^{2}}=\frac{4 \pi n e^{2}}{m_{e} c^{2}}
$$

It is intuitively clear that orbit expansion and charge expulsion are intimately connected: the expanding orbit necessitates that the charge moves outward. However it is not obvious what is the precise quantitative relation, since it will depend on the degree of overlap of the $2 \lambda_{L}$ orbits. How is this relation determined? It turns out that there are several very different paths that lead to exactly the same conclusion. This remarkable coincidence strongly suggests that the result is valid to describe nature.

Let us first state the results. The electric field in the interior of superconductors is caused by a uniform positive charge distribution. For a long cylinder or a sphere, with radius $R$ much larger than the London penetration depth, it is given by

$$
E(r)=E_{m} \frac{r}{R}
$$

and is pointing radially outward. Within a London penetration depth of the surface the electric field is screened by the excess negative charge near the surface and decays to zero at the surface. The maximum electric field, attained for $r \sim R$ is given by 12 ]

$$
E_{m}=-\frac{\hbar c}{4 e \lambda_{L}^{2}}
$$

For $\lambda_{L}=400 A, E_{m}=308,281 \mathrm{~V} / \mathrm{cm}$. Carriers reside in mesoscopic orbits of radius $2 \lambda_{L}$, and move with speed

$$
v_{\sigma}^{0}=\frac{\hbar}{4 m_{e} \lambda_{L}}
$$

with opposite spin electrons moving in opposite directions. Note that

$$
v_{\sigma}^{0}=-\frac{e}{m_{e} c} \lambda_{L} E_{m}
$$

which implies that $v_{\sigma}^{0}$ is also the charge velocity that would be generated by a magnetic field of magnitude $E_{m}$ (in cgs units). The expelled charge density near the surface $\left(\rho_{-}\right)$is related to $E_{m}$ by

$$
E_{m}=-4 \pi \lambda_{L} \rho_{-}
$$

so that the expelled charge density screens the interior electric field. It is also related to the spin current speed $v_{\sigma}^{0}$ by

$$
\rho_{-}=e n_{s} \frac{v_{\sigma}^{0}}{c}
$$

The orbital angular momentum of the carriers in the $2 \lambda_{L}$ orbits moving at speed $v_{\sigma}^{0}$ is

$$
L=m_{e} v_{\sigma}^{0}\left(2 \lambda_{L}\right)=\frac{\hbar}{2} .
$$

\section{MICROSCOPIC DERIVATION}

The spin-orbit interaction derived from Dirac's Hamiltonian for an electron of charge $e$ and mass $m_{e}$ in an electric field $\vec{E}$ is

$$
H_{\text {s.o. }}=-\frac{e \hbar}{4 m_{e}^{2} c^{2}} \vec{\sigma} \cdot(\vec{E} \times \vec{p}) .
$$

We consider the single-particle Hamiltonian

$$
H=\frac{1}{2 m_{e}}\left(\vec{p}-\frac{e}{c} \vec{A}_{\sigma}\right)^{2}
$$

or equivalently

$$
H=\frac{p^{2}}{2 m_{e}}-\frac{e}{m_{e} c} \overrightarrow{A_{\sigma}} \cdot \vec{p}+\frac{e^{2}}{2 m_{e} c^{2}} A_{\sigma}^{2}
$$

with the spin-orbit vector potential $\vec{A}_{\sigma}$ given by $[13]$

$$
\vec{A}_{\sigma}=\frac{\hbar}{4 m_{e} c} \vec{\sigma} \times \vec{E} .
$$

The term linear in $A_{\sigma}$ in Eq. (13b) gives the spin-orbit interaction Eq. (12). In obtaining Eq. (13b) from Eq. (13a), the fact that $\vec{\nabla} \cdot(\vec{\sigma} \times \vec{E})=-\vec{\sigma} \cdot(\vec{\nabla} \times \vec{E})=0$ in an electrostatic situation is used. The significance of the term quadratic in $A_{\sigma}$ in Eq. (13b) will be discussed below.

We propose this Hamiltonian to describe the interaction between the charged superfluid and the compensating ionic background charge. If $n_{s}$ is the density of superfluid carriers of charge $e$, the compensating ionic background has charge density

$$
\rho_{i}=-e n_{s}
$$

The electric field generated by the charge density Eq. (14) in a cylindrical geometry at distance $r$ from the axis is (using Eq. (4))

$$
\vec{E}=-2 \pi e n_{s} \vec{r}=-\frac{m_{e} c^{2}}{2 e \lambda_{L}^{2}} \vec{r}
$$


The spin-orbit vector potential is then

$$
\vec{A}_{\sigma}=-\frac{\hbar c}{8 e \lambda_{L}^{2}}(\vec{\sigma} \times \vec{r})=E_{m} \frac{\vec{\sigma} \times \vec{r}}{2} .
$$

with $E_{m}$ given by Eq. (6). Thus, the superfluid carriers move in a uniform effective magnetic field $\vec{B}_{\sigma}$ given by

$$
\begin{gathered}
\vec{A}_{\sigma}=\frac{\vec{B}_{\sigma} \times \vec{r}}{2} \\
\vec{B}_{\sigma}=E_{m} \vec{\sigma}
\end{gathered}
$$

The radius of the cyclotron motion ('magnetic length') associated with the magnetic field $B_{\sigma}$ in the lowest Landau level is

$$
l_{B_{\sigma}}=\left(\frac{\hbar c}{|e| B_{\sigma}}\right)^{1 / 2}=2 \lambda_{L}
$$

which, as discussed in Sect. II, is the radius of the orbits required to give rise to a Meissner effect. We believe this coincidence is not accidental.

The Hamiltonian term that is quadratic in $A_{\sigma}$ describes the electrostatic energy cost resulting from the orbit expansion and associated charge expulsion. From Eq. (13b), (16) and (4) we obtain

$$
H_{\text {quad }}=\frac{e^{2}}{2 m_{e} c^{2}} A_{\sigma}^{2}=\frac{1}{n_{s}} \frac{E_{m}^{2}}{8 \pi} \frac{r^{2}}{\left(2 \lambda_{L}\right)^{2}}
$$

under the assumption $|\vec{\sigma} \times \hat{n}|=1$, which we can write as

$$
H_{\text {quad }}=\frac{1}{n_{s}} \frac{E_{s}(r)^{2}}{8 \pi}
$$

with

$$
E_{s}(r)=\frac{E_{m}}{2 \lambda_{L}} r .
$$

The electric field $E_{s}(r)$ can be understood as the average electric field resulting from charge expulsion when the orbits expanded to radius $r$, and the Hamiltonian term $H_{\text {quad }}$ is the electrostatic energy density divided by the carrier density $n_{s}$, hence the electrostatic energy cost per carrier.

The existence of $2 \lambda_{L}$ orbits can be understood using a semiclassical argument from the fact that they give rise to minimum total energy, assuming that the angular momentum is fixed at value $\hbar / 2$ (Eq. (11)), which originates in the topological constraint that the pair wavefunction be single-valued. The Hamiltonian Eq. (13b) is, upon replacing $\vec{E}$ by the expression Eq. (15)

$$
H=\frac{p^{2}}{2 m_{e}}+\frac{\hbar}{2 m_{e}} \frac{r}{\left(2 \lambda_{L}\right)^{2}}(\vec{\sigma} \times \hat{n}) \cdot \vec{p}+\frac{\hbar^{2}}{8 m_{e}} \frac{r^{2}}{\left(2 \lambda_{L}\right)^{4}}|\vec{\sigma} \times \hat{n}|^{4} .
$$

For a circular orbit of radius $r$ and angular momentum $\hbar / 2, p=\hbar / 2 r$ and Eq. (22) yields

$H=\frac{\hbar^{2}}{2 m_{e} r^{2}}+\frac{\hbar^{2}}{4 m_{e}\left(2 \lambda_{L}\right)^{2}}(\vec{\sigma} \times \hat{n}) \cdot \hat{p}+\frac{\hbar^{2}}{8 m_{e}} \frac{r^{2}}{\left(2 \lambda_{L}\right)^{4}}|\vec{\sigma} \times \hat{n}|^{4}$.

Assuming $\vec{\sigma}$ is perpendicular to $\hat{n}$ and minimizing Eq. (23) with respect to $r$ yields

$$
r=2 \lambda_{L} .
$$

Thus, the fact that the orbits expand from a microscopic radius to radius $2 \lambda_{L}$ can be understood as driven by lowering of kinetic energy (first term in Eq. (23)) at a cost in potential energy (last term in Eq. (23)) to yield minimal total energy. A similar argument explains why the ground state radius of the electron in a Bohr atom is $a_{0}=\hbar^{2} / m_{e} e^{2}$.

We assume that the single electron states are governed by the Hamiltonian Eq. (22) for the value of $r$ giving minimum energy for the electron orbits, i.e. $r=2 \lambda_{L}$. Hence

$$
H=\frac{p^{2}}{2 m_{e}}+\frac{\hbar q_{0}}{2 m_{e}}(\vec{\sigma} \times \hat{n}) \cdot \vec{p}+\frac{\hbar^{2} q_{0}^{2}}{8 m_{e}}
$$

with $q_{0}=1 / 2 \lambda_{L}$. The eigenstates of Eq. (25) are plane waves, with energy dispersion relation

$$
\epsilon_{k \sigma}=\frac{\hbar^{2} k^{2}}{2 m_{e}}-\frac{\hbar^{2}}{2 m_{e}} q_{0} \vec{k} \cdot(\vec{\sigma} \times \hat{n})+\frac{\hbar^{2} q_{0}^{2}}{8 m_{e}} .
$$

The speed of carriers of spin $\sigma$ and wavevector $\vec{k}$ is

$$
\vec{v}_{\vec{k} \vec{\sigma}}=\frac{1}{\hbar} \frac{\partial \epsilon_{k \sigma}}{\partial \vec{k}}=\frac{\hbar \vec{k}}{m_{e}}-\frac{\hbar q_{0}}{2 m_{e}} \vec{\sigma} \times \hat{n}
$$

so that the carrier's speed increases or decreases by $v_{\sigma}^{0}$ (Eq. (7)) depending on whether $\vec{\sigma} \times \hat{n}$ is parallel or antiparallel to $\vec{k}$. The dispersion relation Eq. (26) gives rise to two Rashba bands

$$
\begin{gathered}
\epsilon_{k}^{1}=\frac{\hbar^{2}}{2 m_{e}}\left(k-\frac{q_{0}}{2}\right)^{2} \\
\epsilon_{k}^{2}=\frac{\hbar^{2}}{2 m_{e}}\left(k+\frac{q_{0}}{2}\right)^{2} .
\end{gathered}
$$

The lowest energy band, $\epsilon_{k}^{1}$, corresponds to spin orientation parallel to $\hat{k} \times \hat{n}$. These bands are shown schematically in Figure 2.

\section{SPIN CURRENT AND KINETIC ENERGY LOWERING}

The motion of carriers with spin current velocity $v_{\sigma}^{0}$ has associated with it a kinetic energy per carrier

$$
\epsilon_{k i n}=\frac{1}{2} m_{e}\left(v_{\sigma}^{0}\right)^{2}
$$




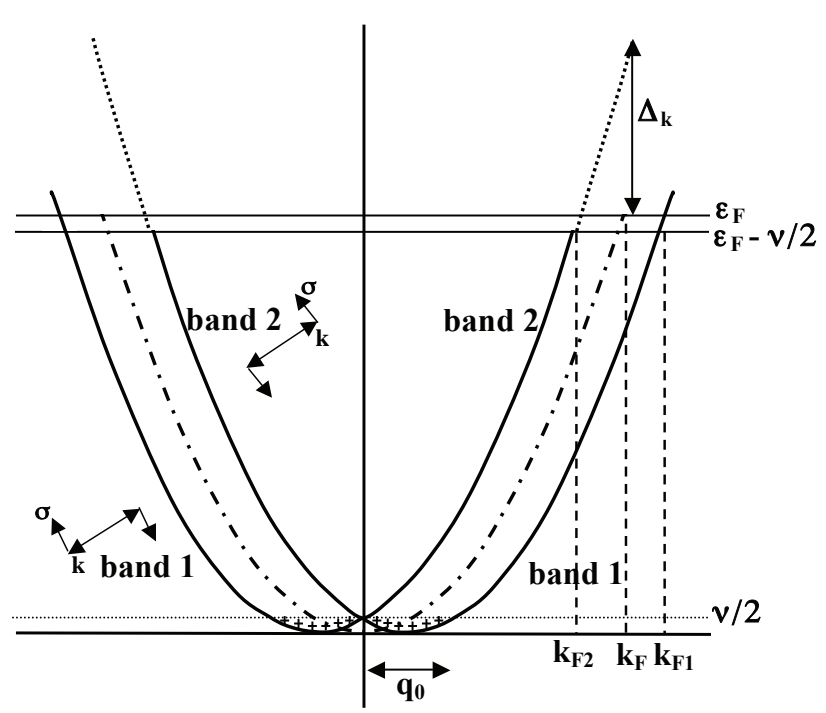

FIG. 2: Rashba bands described by Eq. (28). The Fermi level decreases by $\nu / 2=\hbar^{2} q_{0}^{2} / 8 m_{e}$ relative to the case where $q_{0}=$ 0 . The states in band 1 with $k<q_{0}=1 / 2 \lambda_{L}$ are unoccupied. The direction of the spin $\sigma$ relative to the wavevector is shown schematically for band 1 and band 2 , with the convention that the normal to the closest surface points out of the paper.

so it may seem that generation of a spin current as the system goes superconducting entails an increase in kinetic energy. Remarkably, it is exactly the opposite: the system lowers its kinetic energy as it goes superconducting [14].

One way to see this is as follows: consider a Cooper pair of electrons with opposite spin and spin current velocity $\pm v_{\sigma}^{0}$. When a magnetic field is applied, one of the electrons slows down and the other speeds up by the change in velocity that they acquire, $\Delta v$. The change in kinetic energy of the pair is

$\Delta E=\frac{1}{2} m_{e}\left[\left(v_{\sigma}^{0}+\Delta v\right)^{2}+\left(v_{\sigma}^{0}-\Delta v\right)^{2}\right]-2\left(\frac{1}{2}\left(v_{\sigma}^{0}\right)^{2}\right)=m_{e}(\Delta v)^{2}$

so the kinetic energy increases. When one of the components of the spin current stops, i.e. $\Delta v=v_{\sigma}^{0}$, the system goes normal [10] and at that point the kinetic energy of the pair has increased by $\Delta E=m_{e}\left(v_{\sigma}^{0}\right)^{2}$, from which we conclude that the condensation energy per carrier is

$$
\epsilon_{c}=\frac{1}{2} m_{e}\left(v_{\sigma}^{0}\right)^{2}=\frac{\hbar^{2} q_{0}^{2}}{8 m_{e}}
$$

or in other words, the carriers lower their kinetic energy by $(1 / 2) m_{e}\left(v_{\sigma}^{0}\right)^{2}$, rather than raise it by that amount, as they go superconducting and develop the spin current.

The Rashba bands Eq. (28) describe precisely this physics in a two-dimensional system. The carrier density is given by

$$
n=\frac{k_{F}^{2}}{2 \pi}=\frac{k_{F 1}^{2}}{4 \pi}+\frac{k_{F 2}^{2}}{4 \pi}
$$

where $k_{F}, k_{F 1}, k_{F 2}$ are the Fermi wavevectors in the state without and with spin current (c.f. Fig. 2). Since from Eq. (28) $k_{F 2}=k_{F 1}-q_{0}$, we have $k_{F 1}=\bar{k}_{F}+q_{0} / 2, k_{F 2}=$ $\bar{k}_{F}-q_{0} / 2$, with

$$
\bar{k}_{F}=\sqrt{k_{F}^{2}-q_{0}^{2} / 4}
$$

to satisfy Eq. (32). The Fermi energy is lowered from

$$
\epsilon_{F}=\frac{\hbar^{2} k_{F}^{2}}{2 m_{e}}
$$

to

$$
\epsilon_{F 1,2}=\frac{\hbar^{2} \bar{k}_{F}^{2}}{2 m_{e}}=\epsilon_{F}-\frac{\nu}{2}
$$

with

$$
\nu \equiv \frac{\hbar^{2} q_{0}^{2}}{4 m_{e}} .
$$

For a constant density of states Eq. (35) implies that each carrier lowers its kinetic energy by $\nu / 2$ as the spin current develops in the superconducting state, hence that the condensation energy per electron is $\nu / 2$, in agreement with Eq. (31). This can also be seen directly from the spin orbit interaction Hamiltonian Eq. (12), which gives rise to the spin orbit interaction energy

$$
E_{\text {s.o. }}=\frac{e \hbar}{4 m_{e} c^{2}} \vec{\sigma} \cdot\left(\vec{v}_{\sigma}^{0} \times \vec{E}\right)=\frac{\hbar^{2} q_{0}^{2}}{4 m_{e}}=\nu
$$

for carriers moving with speed $v_{\sigma}^{0}$ given by Eq. (7), and the electric field Eq. (15) evaluated at $r=2 \lambda_{L}$ giving $E=m_{e} c^{2} /|e| \lambda_{L}$. The reason Eq. (37) is twice as large as Eq. (31) is that it does not include the electrostatic energy cost $E_{e l}$

$$
E_{e l}=\frac{1}{n_{s}} \frac{E_{m}^{2}}{8 \pi}=\frac{\nu}{2}
$$

arising from the electrostatic field that develops due to the orbit expansion and associated charge expulsion. In other words, electrons lower their kinetic energy by $\nu$ but give back half of that gain in the electrostatic energy cost associated with charge expulsion and spin current development.

In fact, the density of states associated with the energy dispersion relations Eq. (28) is not constant as would be the case in an ordinary two-dimensional system, rather it is given by (per spin per unit area)

$$
g(\epsilon)=\frac{m_{e}}{2 \pi \hbar^{2}} \frac{k}{\left|k \pm \frac{q_{0}}{2}\right|}
$$

so it is only approximately constant for $k>>q_{0} / 2$. This implies that when the Fermi level drops by $\nu / 2$ the energy lowering per particle is not exactly $\nu / 2$. A direct calculation yields for the change in energy in the presence of spin splitting

$$
\Delta E=N\left[-\frac{\hbar^{2} q_{0}^{2}}{8 m_{e}}+\frac{\hbar^{2} q_{0}^{4}}{48 m_{e} k_{F}^{2}}\right] .
$$


Note that the correction term is very small, a fraction $\sim 10^{-6}$. Nevertheless it has an interesting interpretation. The energy of the electrons in the lower Rashba band in the range $k<q_{0}$ is found to be

$$
E_{\text {core }}=N \frac{\hbar^{2} q_{0}^{4}}{48 m_{e} k_{F}^{2}} .
$$

We have proposed in earlier work [15] that the expelled electrons giving rise to the internal electric field are precisely those in the lower Rashba band with $k<q_{0}$, giving rise to a 'hole core' of unoccupied states (holes) of long wavelength. This is shown schematically in Fig. 2. Since the states are unoccupied their energy has to be substracted from that given by Eq. (40), cancelling the second term in Eq. (40) and thus giving rise to energy lowering per electron of precisely $\nu / 2$, in agreement with Eq. (31).

The holes occupying the bottom of the lower Rashba bands have a 'Fermi surface' at $k=q_{0}$ describing orbits of real space radius $1 / q_{0}=2 \lambda_{L}$ and the associated Fermi velocity is precisely the spin current velocity Eq. (7).

In summary, we have seen in the last two sections that the orbit expansion, kinetic energy lowering, negative charge expulsion and spin current development proposed to take place as a metal undergoes a transition to the superconducting state within the theory of hole superconductivity, can all be understood from the assumption that the magnetic moments of the electrons in the superfluid interact with the compensating positive charge of the ions through the spin orbit interaction resulting from Dirac's Hamiltonian.

\section{SPIN ELECTRODYNAMICS}

The physics described in the previous sections has a simple and consistent description in terms of electrodynamic equations of the same type as London's equations, without any reference to a microscopic Hamiltonian. These equations were derived $[9$, 12] before their microscopic origin was fully elucidated by requiring that the electrodynamic equations be relativistically covariant, and the fact that their predictions coincide with the physics resulting from the microscopic Hamiltonian discussed in the previous section strongly suggest that they describe reality. These equations describe the flow of a spontaneous spin current within a surface layer of thickness $\lambda_{L}$, as shown schematically in Fig. 3 .

In the charge sector, the electrodynamic equations follow from the assumption that the second London equation is valid in the form proposed by London

$$
\vec{J}=-\frac{c}{4 \pi \lambda_{L}^{2}} \vec{A}
$$

with the vector potential $\vec{A}$ obeying the Lorentz rather than the London gauge as in the conventional theory. This is not a statement that violates gauge invariance

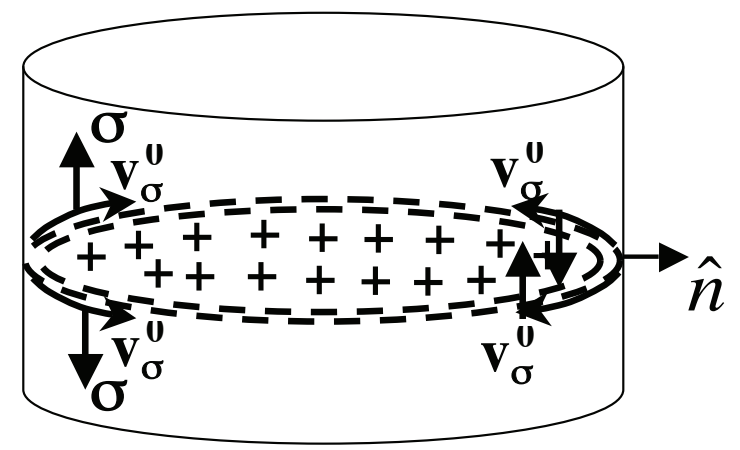

FIG. 3: Schematic depiction of spin current near the surface of a cylindrical superconductor. Superfluid electrons within a London penetration depth of the surface flow counterclockwise (clockwise) if their spin is pointing up (down), with speed $v_{\sigma}^{0}$ given by Eq. (7) .

but a statement about the physics of the system, which adopts a particularly simple form in the gauges just described. Both descriptions of the physics can be expressed in gauge invariant form, and it is only experiment that can ultimately decide which one describes nature.

The electrodynamics in the charge sector is described by the four-dimensional vector equation $[9]$

$$
J-J_{0}=-\frac{c}{4 \pi \lambda_{L}^{2}}\left(A-A_{0}\right)
$$

with

$$
J=(\vec{J}, i c \rho)
$$

$$
A=(\vec{A}, i \phi)
$$

where $\rho$ is the charge density, and $\phi$ the electric potential, and

$$
\begin{aligned}
& J_{0}=\left(0, i c \rho_{0}\right) \\
& A_{0}=\left(0, i \phi_{0}\right) .
\end{aligned}
$$

$\phi_{0}$ is the electric potential originating in the uniform charge distribution $\rho_{0}$ that gives rise to the electric field $E_{m}$ (Eq. (6)) near the surface.

Including spin, the equations are, in a cylindrical geometry with the spin quantization axis parallel to the cylinder axis [12]

$$
J_{\sigma}-J_{\sigma 0}=-\frac{c}{8 \pi \lambda_{L}^{2}}\left(A_{\sigma}-A_{\sigma 0}\right)
$$

with the four-vectors defined as in Eq. (44), with

$$
\begin{gathered}
\vec{A}_{\sigma}=\vec{A}+\lambda_{L} \vec{\sigma} \times \vec{E} \\
\phi_{\sigma}=\phi-\lambda_{L} \vec{\sigma} \cdot \vec{B}
\end{gathered}
$$


and $J=J_{\uparrow}+J_{\downarrow}, \rho=\rho_{\uparrow}+\rho_{\downarrow}, \phi_{\sigma 0}=\phi_{0}$ and

$$
A_{\sigma 0}=\lambda_{L} \vec{\sigma} \times \vec{E}_{0}
$$

where $\vec{E}_{0}(\vec{r})$ is the electric field generated by the uniform charge density $\rho_{0}$ in the interior, that has magnitude $E_{m}$ near the surface. Eq. (47b) follows from Eq. (47a) by requiring that the four-divergence of $A_{\sigma}$ vanishes and using the Lorentz gauge condition.

Note that the electric part of the vector potential $\vec{A}_{\sigma}$ is of the same form as Eq. (13c), with the replacement

$$
r_{q} \equiv \frac{\hbar}{2 m_{e} c} \rightarrow 2 \lambda_{L}
$$

$r_{q}$ is the 'quantum electron radius' [1]. The transition to superconductivity can be understood as an expansion of the electronic wavefunction from the quantum electron radius scale $r_{q}$ to the $2 \lambda_{L}$ scale, keeping the angular momentum fixed at $\hbar / 2$, driven by kinetic energy lowering [14]. The electric field in Eq. (13c) is the bare electric field arising from the charge density $|e| n_{s} \mid$, while the electric field in Eq. (47a) is the net electric field resulting from charge expulsion, which is much smaller. Their ratio is $r_{q} / 2 \lambda_{L}=v_{\sigma}^{0} / c \sim 10^{-6}$.

In the absence of applied magnetic field, Eq. yields for the spin current

$$
\vec{J}_{\sigma}=-\frac{c}{8 \pi \lambda_{L}} \vec{\sigma} \times\left(\vec{E}-\vec{E}_{0}\right) .
$$

In terms of the superfluid density $n_{s}$ and the spin current velocity $\vec{v}_{\sigma}$ we have

$$
\vec{J}_{\sigma}=\frac{e n_{s}}{2} \vec{v}_{\sigma}
$$

The electric field $\vec{E}$ approaches $\overrightarrow{E_{0}}$ in the interior of the superconductor (at distances larger than $\lambda_{L}$ from the surface) and hence the spin current decays to zero in the interior. Near the surface $\vec{E}$ approaches zero as the interior field $\vec{E}_{0}$ is screened by the expelled charge density $\rho_{-}$. At the surface $\vec{E}=0,\left|\vec{E}_{0}\right|=E_{m}$ (Eq. (6)) and Eq. (49) yields for the spin current velocity, using Eq. (4)

$$
\vec{v}_{\sigma}=-\frac{\hbar}{4 m_{e} \lambda_{L}} \vec{\sigma} \times \hat{n}
$$

with the normal unit vector $\hat{n}$ pointing outward. This agrees with the spin current velocity Eq. (27) derived from the microscopic Hamiltonian Eq. (13).

\section{DISCUSSION}

The theory discussed here offers a new conception of the phenomenon of superconductivity, which naturally ties together many well-known aspects of the physics of superconductors in a very different and more fundamental way than the conventional theory does.
Superconductivity in our view results from the expansion of the electronic wavefunctions, driven by kinetic energy lowering. This tendency of quantum particles to expand their spatial range (generally ascribed to the uncertainty principle) can be easily understood semiclassically: an orbiting particle with fixed angular momentum lowers its kinetic energy as the radius of the orbit increases. The need for macroscopic phase coherence follows naturally from the fact that the expanded orbits overlap and thus require phase coherence to avoid collisions that would increase the potential energy. The process of nucleation of the superconducting state in a normal metal matrix naturally leads to the observed physics within our conception: as the orbits expand and the superconducting kernels expands their size, the magnetic field is pushed out of the superconducting regions because of the azimuthal currents induced by the Lorentz force acting on the radially outflowing charge. The charge expulsion (which occurs whether or not a magnetic field is present) naturally leads to the macroscopically inhomogeneous charge distribution depicted in Fig. (1a), as the expelled charge has nowhere to go but the surface if the entire sample becomes superconducting, in the process carrying the expelled magnetic field lines with it. Alternatively, some charge will flow into interior normal regions that trap magnetic field lines, as in the intermediate state of type I superconductors or the mixed (vortex) state of type II superconductors.

The fact that negative charge flows out in the transition to superconductivity has not yet been directly verified experimentally. However I suggest that the phenomenon is clearly illustrated in the current flow through a superconducting wire connected to normal metal leads shown in Fig. 4. Indeed, as the conduction electrons enter the superconducting region they will flow towards the surface as shown by the flow lines in Fig. 4, since current only flows near the surface in superconductors. There is only a small leap to the conclusion that charge flows to the surface when a metal goes superconducting even in

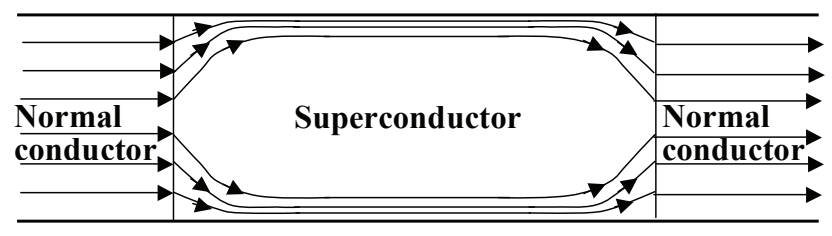

FIG. 4: The current distribution in a superconducting wire which is fed by normal conducting leads. The flow lines are calculated in Ref. [16]. Note that as electrons enter the superconducting region, their velocity acquires a radial component and charge moves towards the surfaces. In the process they carry with them the magnetic field lines (not shown) which exist throughout the interior in the normal leads (circles perpendicular to the plane of the page, with normal in the direction of current flow) and only near the surface (and of course outside the wire) in the superconductor region. 
the absence of current flow [4].

The reader may argue that the sign of the charge moving towards the surface is ambiguous in the situation depicted in Fig. 4, since it depends on the sign of the charge of the current carriers (whether electrons or holes). Fortunately there is no ambiguity: experiments on rotating superconductors demonstrate that the current in superconductors is always carried by negative electrons 6 , $17-$ 19].

Our proposal that superconductors expel negative charge from their interior to the surface was motivated by the theory of hole superconductivity [20], and only later did we realize that it also provides an explanation of the Meissner effect [1], 21 24]. It should be pointed out however that priority for this idea (unbeknownst to this author when refs. [11, 21 24] were written) belongs to K.M. Koch [25], as clearly spelled out in ref.[26]: "Nimmt man nämlich an, dass der Übergang $N \rightarrow S$ in irgendeiner Weise mit einer Elektronenbewegung vom Innern des Versuchskörpers nach seiner Oberflache hin verbunden ist, - und wir werden sofort sehen, dass zur Verwirklichung einer solchen sogar mehrere Möglichkeiten bestehen -, so sieht man ein, dass auf diese Weise ein Abschirmstrom bei konstantem Magnetfeld zustande kommen kann."

The superconducting state envisioned in our theory is 'dynamic', as it involves motion of electrons in mesoscopic orbits (Fig. 1b) and gives rise to a macroscopic spin current near the surface (Fig. 1c). Thus it is closer to the state of "kinetic equilibrium" envisioned by London [27] than the conventional BCS state. The pre-existent spin currents are readily transformed into charge currents when a magnetic field is applied, analogous to the way the "virtual precession" of the electronic angular momentum in an atom is transformed into real precession when a magnetic field is applied [28]. This 'dynamic' ground state is a truly macroscopic quantum state exhibiting quantum zero point motion at the macroscopic level, and it bears a qualitative resemblance to early descriptions of the superconducting state by Bloch, Landau, Frenkel, Smith, Born, Cheng, Heisenberg and Koppe that envisioned domains of charge currents pre-existing in the superconductor in the absence of applied magnetic fields 29 35]. Furthermore, Frenkel 31], Smith 32] and Slater 36] pointed out that electronic orbits of radius of order $\lambda_{L}$ would naturally explain the Meissner effect (this was also unbeknownst to this author when we proposed the existence of $2 \lambda_{L}$ orbits[10]).

In his book 'Superfluids' 16], London coined the term "Meissner pressure". By that term he described the tendency of the superconductor to push out the magnetic field lines, against the "Maxwell pressure" that tries to keep them inside. There is no intuitive physical explanation of "Meissner pressure" within the conventional London-BCS theory of superconductivity however, other than the abstract concept that it originates in the difference in the free energy densities of the normal and superconducting states [16]. Thus, the very descriptive concept of "Meissner pressure" articulated by London remained just that, an appealing physical image with no deeper content. Instead, for us "Meissner pressure" has a concrete physical meaning: it is nothing other than the ubiquitous quantum pressure [37], the tendency of quantum particles to expand their spatial range to lower their kinetic energy, which has as a consequence the outward motion of any interior magnetic field lines as well as the outward motion of negative charge. The 'proximity effect', whereby the superconducting state expands into neighboring normal metal regions, is another vivid manifestation of this physics, as is the prediction that some negative charge 'seeps out' of the surface of superconductors [4, 21].

The tendency of a normal metal to expel negative charge from the interior to the surface and become superconducting will be largest when electronic energy bands have a lot of electrons (almost full bands, resulting in hole-like carriers) and when the conducting structures have excess negative charge (conduction through a network of closely spaced anions). The relevance of these concepts to the understanding of superconductivity in various classes of materials is discussed in ref. [38].
[1] W. Meissner and R. Ochsenfeld, Naturwissenschaften 21, 787 (1933).

[2] M. Tinkham, "Introduction to Superconductivity", 2nd ed, McGraw Hill, New York, 1996.

[3] M.L. Cohen, Mod. Phys. Lett. B 24, 2755 (2010).

[4] J.E. Hirsch, Phys.Rev. B 68, 184502 (2003).

[5] J.E. Hirsch and F. Marsiglio, Physica C 162-164, 591 (1989).

[6] J.E. Hirsch, J. Phys. Chem. Solids 67, 21 (2006).

[7] J.E. Hirsch, Physica C 199, 305 (1992); Physica C 341348, 213 (2000).

[8] J.E. Hirsch and F. Marsiglio, Physica C 331, 150 (2000); Phys.Rev. B 62, 15131 (2000).

[9] J.E. Hirsch. Phys.Rev. B 69, 214515 (2004).

[10] J.E. Hirsch, Europhys. Lett. 81, 67003 (2008).
[11] J.E. Hirsch, J. Sup. Novel Mag. 22, 131 (2009).

[12] J.E. Hirsch, Ann. Phys. (Berlin) 17, 380 (2008).

[13] Y. Aharonov and A. Casher, Phys. Rev. Lett. 53, 319 (1984); J. Anandan, Phys. Lett. A 138, 347 (1989); C.R. Hagen, Phys. Rev. Lett. 64, 2347 (1990); E.M. Chudnovsky, Phys, Rev B 80, 153105 (2009).

[14] J.E. Hirsch, Int. J. Mod. Phys. B 25, 1173 (2011).

[15] J.E. Hirsch, Physica C 470, 635 (2010).

[16] F. London, "Superfluids", Vol. I, Dover, New York, 1950.

[17] J.E. Hirsch. Phys.Rev. B 68, 012510 (2003).

[18] M.A. Sanzari, H.L. Cui and F. Karwacki, Appl. Phys. Lett. 68, 3802 (1996); A.A. Verheijen, J.M. Van Ruitenbeek, R. de Bruyn Ouboter and L.J. de Jongh, 1990 Physica B 165-166 1181 (1990).

[19] L.J. Dunne and T.P. Spiller, J. Phys. Cond. Matt. 4, 
L563 (1992).

[20] J.E. Hirsch, Phys. Lett. A 281, 44 (2001).

[21] J.E. Hirsch, Phys. Lett. A 309, 457 (2003).

[22] J.E. Hirsch, Phys. Lett. A 315, 474 (2003).

[23] J.E. Hirsch, Phys. Lett. A 366, 615 (2007).

[24] J.E. Hirsch, J. Phys. Cond. Matt. 20, 235233 (2008).

[25] K.M. Koch, Z. F. Phys. 116, 586 (1940); Z. F. Phys. 120, 86 (1942); Kolloid-Zeitschrift 105, 89 (1943).

[26] E. Justi and K.M. Koch, Ergeb. der Exact Naturwiss. (Springer Tracts in Mod. Phys.) 21, 117 (1945).

[27] See ref. [16], Introduction.

[28] R.A. Serway, C.J. Moses and C.A. Moyer, "Modern Physics", Third Ed., Thomson Brooks/Cole, Belmont, 2005, p. 299.

[29] F. Bloch, unpublished; referred to in H. Bethe and A.
Sommerfeld, Handbuch der Physik XXIV, p. 12 (1933).

[30] L. Landau, Phys. Zeits. der Sowjet. 4, 43 (1933).

[31] J. Frenkel, Phys. Rev. 43, 907 (1933).

[32] H.G. Smith, University of Toronto Studies, Low Temp. Series Vol. 76, p. 23 (1935); H.G. Smith and J.O. Wilhelm, Rev. Mod. Phys. 7, 237 (935), Sect. 15.

[33] M. Born and K.C. Cheng, Nature 161, 968 (1948); Nature 161, 1017 (1948).

[34] W. Heisenberg, Z. fur Naturf. 2a, 185 (1947); 3a, 65 (1948).

[35] H. Koppe, Ann. der Physik (Leipzig) 1, 405 (1947).

[36] J.C. Slater, Phys, Rev B 52, 214 (1937).

[37] J.E. Hirsch, J. Supercond. Nov. Magn. 23, 309 (2010).

[38] J.E. Hirsch, arXiv:1104.1624 (2011). 